\title{
Risk factors for intraoperative greater trochanteric fractures in hemiarthroplasty for intracapsular femoral neck fractures
}

\author{
Johannes Karl Maria Fakler ${ }^{1}\left[\right.$ - Alexander Brand ${ }^{1}$. Christian Lycke ${ }^{1}$. Christina Pempe ${ }^{1}$. Mohamed Ghanem ${ }^{1}$. \\ Andreas Roth $^{1}$ - Georg Osterhoff ${ }^{1}$. Ulrich Josef Albert Spiegl ${ }^{1}$. Andreas Höch ${ }^{1}$ • Dirk Zajonz ${ }^{1,2,3}$
}

Received: 24 August 2020 / Accepted: 5 November 2020 / Published online: 11 December 2020

(c) The Author(s) 2020

\begin{abstract}
Purpose Hemiarthroplasty is widely accepted as the treatment of choice in elderly patients with a displaced intracapsular femoral neck fracture. Intraoperative greater trochanteric fractures thwart this successful procedure, resulting in prolonged recovery, inferior outcome, and increased risk of revision surgery. Hence, this study analyzed factors potentially associated with an increased risk for intraoperative greater trochanteric fracture.

Methods This retrospective study included 512 hemiarthroplasties in 496 patients with a geriatric intracapsular femoral neck fracture from July 2010 to March 2020. All patients received the same implant type of which $90.4 \%$ were cemented and $9.6 \%$ non-cemented. Intra- and postoperative radiographs and reports were reviewed and particularly screened for greater trochanteric fractures.

Results Female patients accounted for $74 \%$ and mean age of the patients was $82.3( \pm 8.7)$ years. $34(6.6 \%)$ intraoperative greater trochanteric fractures were identified. In relation to patient-specific factors, only a shorter prothrombin time was found to be significantly associated with increased risk of intraoperative greater trochanteric fracture (median 96\%, IQR 82-106\% vs. median $86.5 \%$, IQR $68.8-101.5 \%$; $p=0.046$ ). Other factors associated with greater trochanteric fracture were a shorter preoperative waiting time and changes in perioperative settings. Outcome of patients with greater trochanteric fracture was worse with significantly more surgical site infection requiring revision surgery $(17.6 \%$ vs. $4.2 \%, p=0.005)$.

Conclusion Prolonged prothrombin time, a shorter preoperative waiting time, and implementing new procedural standards and surgeons may be associated with an increased risk of a greater trochanteric fracture. Addressing these risk factors may reduce early periprosthetic infection which is strongly related to greater trochanteric fractures.
\end{abstract}

Keywords Hemiarthroplasty $\cdot$ Femoral neck fracture $\cdot$ Greater trochanteric fracture $\cdot$ Periprosthetic fracture

\section{Introduction}

Hemiarthroplasty (HA) is widely accepted as the treatment of choice in elderly patients with a displaced intracapsular femoral neck fracture [1]. It provides good functional results $[2,3]$ at a fairly low rate of serious complications

Johannes Karl Maria Fakler

johannes.fakler@medizin.uni-leipzig.de

1 Department of Orthopaedic, Trauma and Plastic Surgery, University Hospital of Leipzig, Liebigstr. 20, 04105 Leipzig, Germany

2 Department of Orthopaedic and Trauma Surgery, Zeisigwald Hospitals Chemnitz, Chemnitz, Germany

3 ZESBO-Center for Research on Musculoskeletal Systems, Semmelweisstrasse 14, 04103 Leipzig, Germany requiring unplanned secondary procedures in 3-8\% [2-4]. Intraoperative periprosthetic fractures in hip arthroplasty are specific complications associated with prolonged recovery, inferior outcome, and increased risk of revision surgery [5-7]. The predestinated location of intraoperative iatrogenic fractures is the greater trochanter with an incidence of approximately $3-5 \%$ [8-10]. Although beneficial results after greater trochanteric fractures (GTFs) in individual reports are described [11], persistent pain, a limping gait or significant functional impairment affects $29-65 \%$ of these patients [8-10].

In the fragile group of patients with femoral neck fractures sequelae of GTF might even have more pronounced negative effects. With respect to HA, only limited data are available on intraoperative periprosthetic fractures [12-14]. Data analyzing risk factors specifically for intraoperative 
GTF and their potential effect on outcome are lacking in this group of patients. Subsequently, the primary aim of this study was to identify of risk factors for intraoperative GTF. The secondary aim focused on early outcome parameters in relation to patients without intraoperative GTF.

\section{Methods}

\section{Patient selection and ethical statement}

For this retrospective single-center cohort study, all consecutive patients primarily treated with bipolar HA due to an intracapsular femoral neck fracture were included between July 2010 and March 2020. Patients presenting with a pathologic fracture, prior internal fixation for femoral neck fracture, or presenting with a neglected fracture older than 4 weeks were excluded.

The study was approved by the local Ethics Committee at the University of Leipzig (044/14032016). Written informed consent was obtained from all patients or their legal representative.

\section{Data collection}

Medical charts of all patients were reviewed and demographic data, past medical history, comorbidities, the American Society of Anaesthesiologists (ASA) score, medication, and preoperative routine laboratory blood tests were recorded. From operative reports, description of intraoperative periprosthetic fractures, time and duration of surgery, as well as type of implants were documented.

Median follow-up was $6(0-23)$ months and a minimum follow-up of 30 days or until death within 30 days was available in $77.1 \%$ of all HAs.

\section{Surgical treatment}

Indications for HA were made in accordance with national guidelines. Surgery was performed in general anesthesia and all patients received a single-shot preoperative antibiotic prophylaxis with cefuroxime or clindamycin in case of history of allergy to penicillin. Patients were placed in a supine position. A modified Hardinge approach was applied in $90.8 \%$ and an anterolateral approach in 9.2\%. A bipolar hemiprosthesis (Corail stem, bipolar metal head, DePuy Synthes, Warsaw, USA) was the standard implant at our institution. Cemented and non-cemented stems were used in $90.4 \%$ and $9.6 \%$, respectively. Because the stem design and corresponding rasp were demonstrated to influence occurrence of intraoperative periprosthetic fractures [13], we excluded 11 HAs that sporadically were performed with other implants than the Corail stem. Regular day time surgical service was provided from 8 a.m. to
8 p.m., on-call service from 8 p.m. to 8 a.m. Postoperatively, patients were mobilized with full weight-bearing provided that they were ambulatory.

49 surgeons performed a hemiarthroplasty in these patients during the observed period of time. Surgeons that performed at least 100 elective hip replacements and continued to be involved in elective hip replacement were defined as hip arthroplasty surgeons (HAS).

Until 2014, hip hemiarthroplasty for intracapsular femoral neck fracture was performed by orthopedic trauma surgeons. In 2014 , the formerly separated departments of trauma orthopedic surgery and (elective) orthopedic surgery joined together. Since then, orthopedic surgeons, which primarily were involved in elective hip replacements, were engaged in operative treatment of trauma patients. These surgeons used different hip arthroplasty implants for elective hip replacement before 2014. In 2016, a certified center of arthroplasty was established requiring implementation of additional quality standards with reference to clinical pathways, qualification of surgeons and quality management.

\section{Radiographic evaluation}

Preoperative, intraoperative, as well as postoperative standard anteroposterior pelvis and axial hip radiographs were analyzed by an experienced senior surgeon. All identified greater trochanteric fractures were confirmed by a second experienced senior surgeon. Preoperative and postoperative radiographs were available for review in $100 \%$ and $99 \%$, respectively.

\section{Statistical analysis}

Continuous variables were analyzed for normal distribution applying the Kolmogorov-Smirnov test in addition to $\mathrm{q}-\mathrm{q}$ diagrams. Normally distributed parameters were given as mean and standard deviation (SD); for non-normally distributed parameters, median and the interquartile range [25th-75th percentile] were used. Continuous comparisons were performed using the $t$ test for normally distributed variables and the Mann-Whitney $U$ test for non-normally distributed variables. For categorical comparisons, according to group size, Chi-squared or Fisher's test were performed. All statistical computations were performed using SPSS version 24.0 (Chicago, IL, USA). $p$ values less than $5 \%$ were considered as significant.

\section{Results}

496 patients with 512 intracapsular femoral neck fractures were treated with HA and met inclusion criteria. Female patients accounted for $74 \%$ and mean age of the patients 
was $82.3( \pm 8.7)$ years. 30 -day mortality was $8.8 \%$. During the inclusion period from 2010 to 2020 , number of patients, mean patient age, and body mass index (BMI) remained constant (Fig. 1). Surgery was performed on call in $18.9 \%$ and within $24 \mathrm{~h}$ in $48.4 \%$ of all HAs.

Operative reports as well as intraoperative and postoperative radiographs revealed 34 GTFs (6.6\%). Only one GTF was fixed intraoperatively by a cerclage wire; no secondary procedures for greater trochanteric fixation were performed.

To identify risk factors, the GTF group was compared to controls without isolated fracture of the greater trochanter. With regard to patient-related factors, only prothrombin time was found to be significantly lower in the GTF group. All other patient-related parameters displayed no differences between groups (Table 1).

Moreover, risk factors associated with surgery and perioperative settings were analyzed. Preoperative waiting time was significantly shorter in the GTF group (19 h vs $26 \mathrm{~h}$; $p=0.006)$. Most of the HA were performed by attending surgeons in both groups $(75 \%$ vs. $71 \%, p=0.683)$. Although the fraction of hip arthroplasty surgeons (HAS) was lower in the GTF group compared to controls, this difference was not significant ( $27 \%$ vs. $38 \%, p=0.203) .347$ HAs were performed before and 165 HAs after the establishment of a center of arthroplasty at our department with implementation of additional quality standards. Accordingly, only $31 \%$ of HAs in the control group with no GTF accounted for the younger period of the hip arthroplasty center. However, number of GTFs distributed equally between these periods with $50 \%$ in each (31\% vs. $50 \%, p=0.046)$ (Table 2$)$. The
Table 1 Patient-related factors associated with risk of intraoperative greater trochanteric fracture (GTF)

\begin{tabular}{llll}
\hline & $\begin{array}{l}\text { Controls } \\
\text { (no GTFx, } n=478)\end{array}$ & $\begin{array}{l}\text { GTFx } \\
(n=34)\end{array}$ & $p$ \\
\hline Age (years) & $82.4 \pm 8.6$ & $81.1 \pm 10.0$ & 0.471 \\
Female sex & $357(75 \%)$ & $24(71 \%)$ & 0.684 \\
BMI & $24.4(22.0-27.0)$ & $23.5(22.0-26.8)$ & 0.546 \\
Platelet inhibitors & $157(33 \%)$ & $9(27 \%)$ & 0.460 \\
Oral anticoagulants & $84(18 \%)$ & $7(21 \%)$ & 0.817 \\
Warfarin & $44(9 \%)$ & $5(15 \%)$ & 0.358 \\
NOAKs & $40(8 \%)$ & $2(6 \%)$ & 0.759 \\
Diabetes & $129(27 \%)$ & $7(21 \%)$ & 0.472 \\
Smoking & $58(12 \%)$ & $1(3 \%)$ & 0.160 \\
Oral glucocorticoids & $29(6 \%)$ & $0(0 \%)$ & 0.246 \\
CRP (mg/l) & $5.6(1.6-24.7)$ & $7.7(1.9-28.0)$ & 0.622 \\
Leucocyte count & $10.2(8.0-13.2)$ & $11.4(8.6-15.1)$ & 0.149 \\
$\quad($ exp 9/l) & & & \\
25OH D3 (ng/ml) & $8.0(5.0-13.1)$ & $5.2(4.0-13.1)$ & 0.194 \\
Creatinine $(\mu \mathrm{mol} / \mathrm{l})$ & $80.0(64.0-104.0)$ & $77.5(64.0-106.5)$ & 0.770 \\
Hemoglobin & $7.7(6.9-8.4)$ & $7.7(6.8-8.4)$ & 0.878 \\
$\quad$ (mmol/l) & & $86.5(68.8-101.5)$ & 0.046 \\
Prothrombin time & $96(82-106)$ & & 0.547 \\
$\quad$ (\%) & & $27(79 \%)$ & \\
ASA 3 and 4 & $352(74 \%)$ & & \\
\hline
\end{tabular}

Values are given as mean ( \pm standard deviation), median (IQR) or absolute numbers and percentage. BMI body mass index, NOAKs non-vitamin K-dependent oral anticoagulants, CRP c-reactive protein, 25OH D3 25-hydroxy vitamin D3

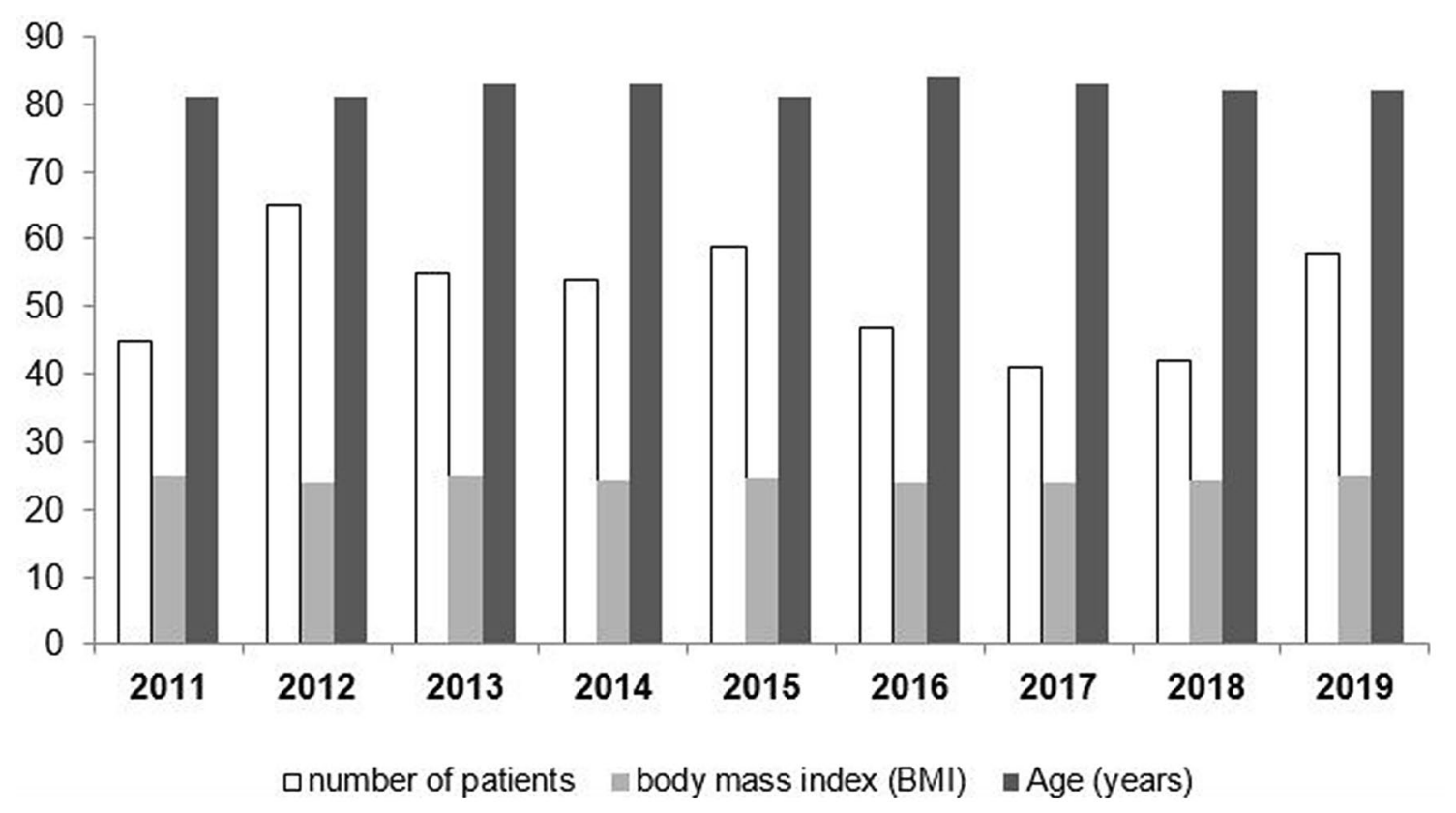

Fig. 1 Anual case load and patient characteristics during study period 
number of different orthopedic surgeons was 38 before and 29 after implementing the hip arthroplasty center. Nevertheless, the average case load was substantially higher in the earlier period with 9.1 compared to 5.7 HAs per surgeon.

Patients suffering an intraoperative GTF noticeably affected early postoperative results to the worse (Table 3 ). Length of hospital stay (LOS) was longer and the rate of postoperative hematoma and seroma requiring further revision surgery tended to be higher $(8.8 \%$ vs. $2.5 \%, p=0.070)$. Finally, early deep infections were substantially higher in the GTF group (17.6\% vs. $4.2 \%, p=0.005)$.

\section{Discussion}

Our retrospective study found $34(6.6 \%)$ isolated GTFs which is in line with other studies that found GTFs in $4.1-6.3 \%$ following HA in patients with a femoral neck fracture [12-14]. The only patient-related risk factor for intraoperative GTF identified in our study was a decreased prothrombin time. Although more patients were on warfarin in the GTF group, the difference was not significant $(15 \%$ vs. $9 \%, p=0.358)$. However, prothrombin time may have also been influenced by vitamin $\mathrm{K}$ deficiency related to malnutrition or medical disease. Vitamin K plays an important role in bone health and is highly prevalent in patients with hip fracture $[15,16]$. In addition, 25OH-vitamin D3 levels tended to be lower in the GTF group supporting the hypothesis that reduced bone health may result in increased susceptibility to iatrogenic fractures. Otherwise, a lower prothrombin time may indicate increased bleeding intraoperatively, especially during rasping the intramedullary canal, which may limit a clear view and urge the surgeon to conclude surgery promptly. This in turn increases the stress level of the surgeon, potentially altering performance with a higher rate of GTFs.

In contrast to our study, no differences of $25 \mathrm{OH}$-vitamin D3 levels were found between patients suffering an intraoperative periprosthetic fracture compared to patients without fracture in a study of 271 treated with HA for femoral neck fracture [12]. However, patients in this study presented with mean 25OH-vitamin D3 levels of $20 \mathrm{ng} / \mathrm{ml}$, whereas hip fracture patients in our region exhibit significantly lower levels with a median of $8.4 \mathrm{ng} / \mathrm{ml}$ as shown previously [17]. Hong et al. found no difference of T-Scores between groups, but identified Dorr C type femoral canals [18] as a risk factor for intraoperative fractures as did Bellova et al. [14]. Since we focused on isolated GTF, the Dorr types of the femoral canals were not determined. Bellova et al. [14] found female sex as a risk factor for intraoperative iatrogenic fractures in their series of $481 \mathrm{HA}$. We and Hong et al. [12] could not confirm sex as a risk factor.

Preoperative waiting time was significantly shorter in the GTF group. Shorter preoperative waiting times may limit optimal preparation and assembly of a well-rehearsed team. Although not significantly, HAS were less frequently involved in the GTF group compared to controls in our study. Moreover, $51.6 \%$ of all patients were operated beyond $24 \mathrm{~h}$ after admission and $81.1 \%$ during routine daytime hours at our institution-which more likely allows allocation of a suitable specialized team. However, surgery performed
Table 2 Surgical and perioperative factors associated with risk of intraoperative greater trochanteric fracture (GTF)

\begin{tabular}{llll}
\hline & $\begin{array}{l}\text { Controls } \\
(\text { no GTFx, } n=478)\end{array}$ & $\begin{array}{l}\text { GTFx } \\
(n=34)\end{array}$ & $p$ \\
\hline Preoperative time (h) & $26(16-42)$ & $19(9-30)$ & $0.006^{*}$ \\
Duration surgery (min)* & $71(57-84)$ & $75(61-92)$ & 0.142 \\
Cemented & $430(90 \%)$ & $33(97 \%)$ & 0.235 \\
Surgery on call & $91(19.0 \%)$ & $6(17.6 \%)$ & 1.000 \\
Attending surgeon & $359(75 \%)$ & $24(71 \%)$ & 0.683 \\
Hip arthroplasty surgeon (HAS) & $181(38 \%)$ & $9(27 \%)$ & 0.203 \\
Established center of arthroplasty & $148(31 \%)$ & $17(50 \%)$ & $0.024^{*}$ \\
\hline
\end{tabular}

Values are given as median (IQR) or absolute numbers and percentage, $* \mathrm{p}<0.05$

\begin{tabular}{llll}
\hline & $\begin{array}{l}\text { Controls } \\
\text { (no GTFx, } n=478)\end{array}$ & $\begin{array}{l}\text { GTFx } \\
(n=34)\end{array}$ & $p$ \\
\hline Length of stay (LOS) & $10.0(8.0-14.0)$ & $12.5(9.9-16.5)$ & $0.009^{*}$ \\
30-day mortality & $43(9.0 \%)$ & $2(5.9 \%)$ & 0.757 \\
Revision surgery due to hematoma/seroma & $12(2.5 \%)$ & $3(8.8 \%)$ & 0.070 \\
Revision surgery due to early deep infection & $20(4.2 \%)$ & $6(17.6 \%)$ & $0.005^{*}$ \\
\hline
\end{tabular}

Values are given as median (IQR) or absolute numbers and percentage, $* \mathrm{p}<0.05$ 
on-call had no influence on GTF as confirmed by others [14].

Although not by significance, highly specialized hip arthroplasty surgeons (HAS) were represented less frequently in the GTF group than in the control group, implicating that surgical experience may be associated with favorable outcome with regard to this specific complication. Nevertheless, no reduction of GTF was observed after establishment of a certified arthroplasty center, despite implementation of additional quality standards with reference to clinical pathways, qualification of surgeons, and quality management. $50 \%$ of the GTFs were recorded after implementation of our arthroplasty center, whereas in the non-fracture group, only $31 \%$ of HAs were performed in the period of the arthroplasty center. An explanation could be that by restructuring the arthroplasty service new surgeons had to be integrated into acute fracture arthroplasty. They had to adapt to hip implants and instruments specifically used for acute HAs in femoral neck fracture patients, but not for elective total hip arthroplasty at our institution. In addition, a significantly lower number of HAs was implanted during the considerably shorter period of the hip arthroplasty center underlined by a substantially lower case load per surgeon. Moreover, an implant or instrumentation (i.e., rasp) immanent issue might be responsible for the relatively high rate of GTF in this period. This is supported by Laflamme et al. [13] who found an abnormal number of iatrogenic intraoperative fractures following HA for femoral neck fractures after they switched to the same implant inserted at our institution. They observed 22 (6.3\%) iatrogenic GTFs in 348 HAs which is almost identical to our results. Other factors potentially affecting the risk of GTF, as, for example, femoral offset influenced by implant size and design, were not analyzed in our study. However, Laflamme et al. [13] found no relationship between implant sizes and GTF. Moreover, only limited information was found in our operation reports regarding the stage of the surgery at what a GTF occurred. On the other side, it is not always possible to differentiate exactly, whether a GTF originated during hip adduction to expose the proximal femoral canal, preparation of the femoral canal, stem insertion, or reduction of the hip after implantation.

Patients with GTFs after THA were demonstrated to experience a worse outcome with limping, increased pain, and functional impairment [8-10]. In addition, Homma et al. [19] hypothesized that even minor GTFs with a small fragment (chip fracture) may result in increased bleeding and formation of hematoma. This is confirmed in our study. Seroma and hematoma requiring surgical revision clearly tended to be more frequently after GTFs. A significant association was shown between GTFs and early postoperative periprosthetic infections requiring revision.

This study is limited by its retrospective design including patients over a long period of time. Thus, it may be subject to changes and confounders not identified. However, annual case load and patient characteristics remained constant (Fig. 1). Another limitation may be that beyond postoperative radiographs usually no additional or routine follow-up radiographs were ordered and thus not considered. Thus, non-displaced GTFs not identified on postoperative radiographs, but dislocating secondarily may have been missed. Further limitations may be seen in the unequal distribution of the two different approaches used as well as not addressing anatomical aspects of the proximal femur (i.e., varus or valgus hip) which may also have an impact on risk of GTF. Finally, the number of patients with a GTF is relatively small compared to the non-fracture group potentially impairing statistical analysis. The strength of the study lies in a high number of patients that received the same type of implant.

\section{Conclusion}

Prolonged prothrombin time, a shorter preoperative waiting time, and implementing new procedural standards and surgeons may be associated with an increased risk of a GTF. Addressing these risk factors may improve outcome, especially by reducing early periprosthetic infection which is strongly related to GTFs.

Funding Open Access funding enabled and organized by Projekt DEAL.

\section{Compliance with ethical standards}

Conflict of interest Johannes KM Fakler, Alexander Brand, Christian Lycke, Christina Pempe, Mohamed Ghanem, Andreas Roth, Georg Osterhoff, Ulrich JA Spiegl, Andreas Höch, and Dirk Zajonz declare that they have no conflict of interest.

Open Access This article is licensed under a Creative Commons Attribution 4.0 International License, which permits use, sharing, adaptation, distribution and reproduction in any medium or format, as long as you give appropriate credit to the original author(s) and the source, provide a link to the Creative Commons licence, and indicate if changes were made. The images or other third party material in this article are included in the article's Creative Commons licence, unless indicated otherwise in a credit line to the material. If material is not included in the article's Creative Commons licence and your intended use is not permitted by statutory regulation or exceeds the permitted use, you will need to obtain permission directly from the copyright holder. To view a copy of this licence, visit http://creativecommons.org/licenses/by/4.0/.

\section{References}

1. Bhandari M, Devereaux PJ, Tornetta P, Swiontkowski MF, Berry DJ, Haidukewych G, et al. Operative management of displaced femoral neck fractures in elderly patients. An international survey. J Bone Joint Surg Am. 2005;87(9):2122-30. 
2. HEALTH Investigators, Bhandari M, Einhorn TA, Guyatt G, Schemitsch EH, Zura RD, Sprague S, Frihagen F, Guerra-Farfán E, Kleinlugtenbelt YV, Poolman RW, Rangan A, Bzovsky S, Heels-Ansdell D, Thabane L, Walter SD, Devereaux PJ. Total hip arthroplasty or hemiarthroplasty for hip fracture. N Engl J Med. 2019;381(23):2199-208. https://doi.org/10.1056/NEJMo a1906190.

3. Chammout G, Kelly-Pettersson P, Hedbeck CJ, Stark A, Mukka S, Sköldenberg O. HOPE-trial: hemiarthroplasty compared with total hip arthroplasty for displaced femoral neck fractures in octogenarians: a randomized controlled trial. JB JS Open Access. 2019;4(2):e0059. https://doi.org/10.2106/JBJS.OA.18.00059.

4. Bartels S, Gjertsen JE, Frihagen F, Rogmark C, Utvåg SE. High failure rate after internal fixation and beneficial outcome after arthroplasty in treatment of displaced femoral neck fractures in patients between 55 and 70 years. Acta Orthop. 2018;89(1):53-8. https://doi.org/10.1080/17453674.2017.1376514 ((Epub 2017 Sep 15))

5. Kahn A, O'Driscoll M. Fractures of the femur during total hip replacement and their management. J Bone Joint Surg Br. 1977;59:36-41.

6. Thillemann TM, Pedersen AB, Johnsen SP, Soballe K. Inferior outcome after intraoperative femoral fracture in total hip arthroplasty. Act Orthopaedica. 2008;79:327-34.

7. Sheth NP, Brown NM, Moric M, Berger RA, Della Valle CJ. Operative treatment of early peri-prosthetic femur fractures following primary total hip arthroplasty. J Arthroplasty. 2013;28(2):286-91. https://doi.org/10.1016/j.arth.2012.06.003 ((Epub 2012 Aug 3)).

8. Hendel D, Yasin M, Garti A, Weisbort M, Beloosesky Y. Fracture of the greater trochanter during hip replacement: a retrospective analysis of 21/372 cases. Acta Orthop Scand. 2002;73(3):295-7.

9. Pritchett JW. Fracture of the greater trochanter after hip replacement. Clin Orthop Relat Res. 2001;390:221-6.

10. Brun OC, Maansson L. Fractures of the greater trochanter following total hip replacement. Hip Int. 2013;23(2):143-6. https://doi. org/10.5301/HIP.2013.10813.

11. Probst A, Wetterkamp D, Neuber M. Iatrogenic avulsion of the greater trochanter during prosthetic replacement of the hip. [Article in German]. Unfallchirurg. 1999;102(6):497-9.
12. Hong CC, Nashi N, Tan JH, Manohara R, Lee WT, Murphy DP. Intraoperative periprosthetic femur fracture during bipolar hemiarthroplasty for displaced femoral neck fractures. Arch Orthop Trauma Surg. 2018;138(9):1189-98. https://doi.org/10.1007/ s00402-018-2952-7 ((Epub 2018 May 17)).

13. Laflamme M, Angers M, Vachon J, Pomerleau V, Arteau A. High incidence of intraoperative fractures with a specific cemented stem following intracapsular displaced hip fracture. J Arthroplasty. 2020;35(2):485-9. https://doi.org/10.1016/j.arth.2019.09.017 ((Epub 2019 Sep 17)).

14. Bellova P, Baecker H, Lotzien S, Brandt M, Schildhauer TA, Gessmann J. Risk analysis and clinical outcomes of intraoperative periprosthetic fractures: a retrospective study of 481 bipolar hemiarthroplasties. J Orthop Surg Res. 2019;14(1):432. https:// doi.org/10.1186/s13018-019-1494-1.

15. Rodríguez-Olleros RC, Díaz CM. Vitamin K and bone health: a review on the effects of vitamin K deficiency and supplementation and the effect of non-vitamin $\mathrm{K}$ antagonist oral anticoagulants on different bone parameters. J Osteoporos. 2019;2019:2069176. https://doi.org/10.1155/2019/2069176.

16. Nakano T, Tsugawa N, Kuwabara A, Kamao M, Tanaka K, Okano $\mathrm{T}$. High prevalence of hypovitaminosis $\mathrm{D}$ and $\mathrm{K}$ in patients with hip fracture. Asia Pac J Clin Nutr. 2011;20(1):56-61.

17. Fakler JK, Grafe A, Dinger J, Josten C, Aust G. Perioperative risk factors in patients with a femoral neck fracture-influence of 25-hydroxyvitamin D and C-reactive protein on postoperative medical complications and 1-year mortality. BMC Musculoskelet Disord. 2016;17:51. https://doi.org/10.1186/s12891-016-0906-1.

18. Dorr LD, Faugere MC, Mackel AM, Gruen TA, Bognar B, Malluche HH. Structural and cellular assessment of bone quality of proximal femur. Bone. 1993;14(3):231-42. https://doi. org/10.1016/8756-3282(93)90146-2.

19. Homma Y, Baba T, Ochi H, Ozaki Y, Kobayashi H, Matsumoto M, Yuasa T, Kaneko K. Greater trochanter chip fractures in the direct anterior approach for total hip arthroplasty. Eur J Orthop Surg Traumatol. 2016;26(6):605-11. https://doi.org/10.1007/ s00590-016-1798-3. 